\title{
Fast Load Control with Stochastic Frequency Measurement
}

\author{
Changhong Zhao, Ufuk Topcu, Member, IEEE, and Steven H. Low, Fellow, IEEE
}

\begin{abstract}
Matching demand with supply and regulating frequency are key issues in power system operations. Flexibility and local frequency measurement capability of loads offer new regulation mechanisms through load control. We present a frequency-based fast load control scheme which aims to match total demand with supply while minimizing the global end-use disutility. Local frequency measurement enables loads to make decentralized decisions on their power from the estimates of total demand-supply mismatch. To resolve the errors in such estimates caused by stochastic frequency measurement errors, loads communicate via a neighborhood area network. Case studies show that the proposed load control can balance demand with supply and restore the frequency at the timescale faster than AGC, even when the loads use a highly simplified system model in their algorithms. Moreover, we discuss the tradeoff between communication and performance, and show with experiments that a moderate amount of communication significantly improves the performance.
\end{abstract}

Index Terms-Power system control, communication, load control, distributed control, optimization

\section{INTRODUCTION}

In power systems, the mismatch between load and generation must be corrected within short time, otherwise it will cause frequency to deviate from the normal level, threaten system stability, or more seriously, cause permanent damage to facilities [1]. Hence, matching load with supply and regulating frequency have been important goals in power system control.

To serve these goals, conventional efforts focus on the generation side. For example, the automated generation control (AGC) issues signals to control reserved generation and minimize the area control error (ACE) composed of frequency deviation and unscheduled tie-line power flows [2]. In addition, generators are equipped with speed governors that adjust the mechanical power proportionally to the frequency deviation [3],[4]. Other generation control components include the automatic voltage controller and the power system stabilizer, etc. [5]. However, relying solely on regulating generation is sometimes inefficient. For example, the AGC takes as long as 5-15 minutes to drive the ACE to its setpoint [2], and the reserved generators must be grid connected and part-loaded, increasing the cost and emissions [6].

A lot of works have also investigated the effect of load participation in power system control, especially the frequencybased load control. For example, Brooks et al. state that

This work is supported by NSF NetSE grants CNS 0911041, Southern California Edison (SCE), Okawa Foundation, Boeing Corporation, and Cisco.

C. Zhao, U. Topcu and S. H. Low are with the Division of Engineering and Applied Science, California Institute of Technology, Pasadena, CA 91125, USA. e-mail: (czhao@caltech.edu, utopcu@cds.caltech.edu, slow@caltech.edu). loads can sense the frequency as a measure of supply-demand mismatch and respond accordingly in less than 1 second [7]. The Grid Friendly Appliance Controller [8] developed by the PNNL suggests that appliances can provide fast reserve within seconds by responding to certain trends in the frequency. Trudnowski et al. assumed the loads can be adjusted proportionally to frequency deviations, and investigated multiple issues such as the distribution of loads, time delay and discretized load action [3]. Our previous work in [10] suggested a load control scheme where the loads respond to local frequency measurements, match total demand with supply and minimize global end-use disutility in a decentralized way. All the works above show that the frequency-based load control is fast and makes the communication between loads and a central coordinator no longer essential, thus decentralizing the control.

The decentralized scheme we proposed in [10] performs poorly in the presence of stochastic frequency measurement errors at individual loads. In this paper, we design a new algorithm where the loads communicate via the neighborhood area network to jointly compensate for the effect of stochastic errors. We prove that the change of loads converges to the solution of the same global optimization problem as in [10] under the new algorithm. In our scheme each load estimates the global demand-supply mismatch using a model of the power system. Numerical experiments show that the proposed scheme is robust to inaccuracies in the power system model, and hence more suitable for practical use. The experiments also show the tradeoff between the inter-load communication effort and the performance of the load control scheme.

This paper is organized as follows. Section II describes the power system model and the load control optimization problem. Section III introduces the approach of estimating total demand-supply mismatch from local frequency measurement. Section IV presents the decentralized load control algorithm and proves its convergence. Section $\mathrm{V}$ shows the results of case studies. Finally, Section VI provides concluding remarks, suggests limitations and gives outlook of future studies.

\section{PRoblem SETUP}

\section{A. System model}

Fig. 1 shows the schematic diagram of the power system under consideration. It contains the generation, transmission, distribution system and controllable loads within a strongly connected area [2], in which we consider the dynamics of a single synchronous machine and ignore the asynchronism across multiple machines. Let $V=\{1,2, \ldots N\}$ denote the set of loads. Each load $i \in V$ consumes real power $d_{i}$ which 


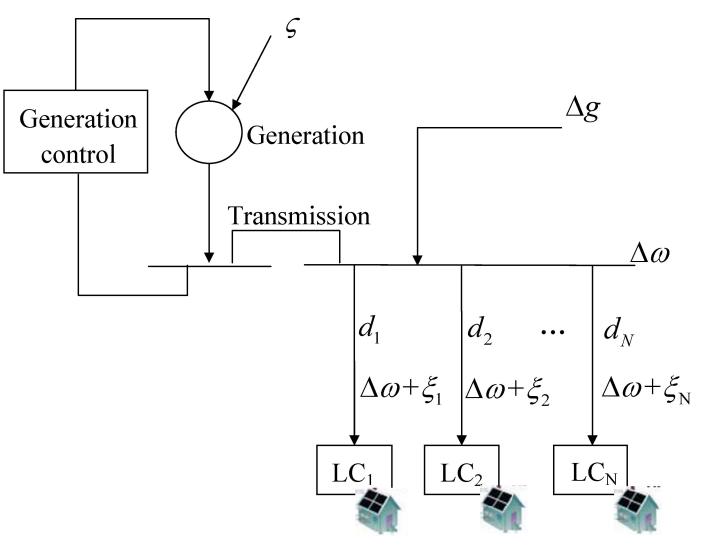

Fig. 1. Schematic diagram of the system under consideration.

is regulated by a controller $L C_{i}$. An unscheduled change in real power supply is denoted by $\Delta g$. For example, $\Delta g$ may be unscheduled tie-line power flow, a sudden peak in load, or a sudden generation loss. There exists some stochastic disturbance $\zeta$ in the system process, which may come from renewable generation, unit tripping, change in grid facilities or change in system parameters caused by the environment.

At time $t$, the load controller $L C_{i}$ measures the frequency deviation $\Delta \omega_{t}$ from the nominal value (e.g., $60 \mathrm{~Hz}$ ), and calculates the change in load $\Delta d_{i}(t)$ accordingly. Let $\Delta d_{i}(t)>0$ stand for decreasing the load. Then the total load change is $\Delta d(t)=-\sum_{i \in V} \Delta d_{i}(t)$. Define the total demand-supply mismatch at time $t$ as $u_{t}:=\Delta d(t)-\Delta g(t)$. Then we have a system model that characterizes the relation between $\Delta \omega_{t}$ and $u_{t}$. As is a usual approach to analyze the power system stability [9], the system is described by a transfer function $G(s)$ such that $\Delta \omega(s)=G(s) u(s)$. The load control scheme we are to propose is a sampled control system, i.e., loads sample $\Delta \omega$ and make decisions once every $\Delta t$ time. Hence, we use $t=0,1,2, \ldots$ instead of $t=0, \Delta t, 2 \Delta t, \ldots$ to denote the time, and a discretetime power system model

$$
\begin{cases}x_{t+1} & =A x_{t}+B u_{t}+\zeta_{t} \\ \Delta \omega_{t} & =C x_{t}\end{cases}
$$

where $x_{t} \in \mathbb{R}^{n}$ is the state vector, $u_{t} \in \mathbb{R}$ is the total demandsupply mismatch and $\zeta_{t} \in \mathbb{R}^{n}$ is the process disturbance, all at time $t$. The matrices $A \in \mathbb{R}^{n \times n}, B \in \mathbb{R}^{n \times 1}$, and $C \in \mathbb{R}^{1 \times n}$. We assume $C B \neq 0$, whose justification is discussed in [10].

At time $t$, load $i$ measures the frequency deviation $\Delta \omega_{t}$, and gets a measurement $\Delta \bar{\omega}_{i, t}=\Delta \omega_{t}+\xi_{i, t}$, which differs from the true deviation $\Delta \omega_{t}$ by a stochastic error $\xi_{i, t}$. We assume both $\zeta_{t}$ and $\xi_{i, t}$ have zero mean, and for all $t, s \geq 0$ and all $i, j \in V$, their covariances satisfy

$$
\mathbb{E}\left(\zeta_{t} \zeta_{s}^{T}\right)=Q \delta_{t s}, \mathbb{E}\left(\xi_{i, t} \xi_{j, s}\right)=W \delta_{t s} \delta_{i j}
$$

where $Q \in \mathbb{R}^{n \times n}$ is a positive semi-definite matrix, $W \geq 0, \delta_{t s}$ and $\delta_{i j}$ are both the Kronecker delta, and $\mathbb{E}$ is the expectation operator.

\section{B. Load control optimization problem}

We now present the optimal load control problem. It is the same as what we formulated in [10], which aims to match the change in demand with the change in supply, i.e., to drive $u_{t}$ to zero, and minimize the global disutility caused by changing the power consumption of loads. By $\Delta \omega(s)=G(s) u(s)$, the frequency deviation will also be driven to zero if $u_{t}$ is driven to zero.

We consider the static case where the change in supply $\Delta g$ is time-invariant, i.e., $\Delta g(t)=\Delta g$ for all $t \geq 0{ }^{1}$, and without loss of generality, assume $\Delta g<0$. Each load $i$ has a disutility $D_{i}\left(\Delta d_{i}\right)$, where $\Delta d_{i} \in\left[0, \bar{d}_{i}\right]$ and $\bar{d}_{i}$ is the maximum change in load $i$ allowed by appliance design or user permission. The load control optimization problem, denoted by $\mathbf{P P}$, is presented as

$$
\mathbf{P P} \begin{cases}\min _{\Delta d_{i} \in\left[0, \bar{d}_{i}\right]} & \sum_{i=1}^{N} D_{i}\left(\Delta d_{i}\right) \\ \text { subject to } & -\sum_{i=1}^{N} \Delta d_{i}-\Delta g=0 .\end{cases}
$$

For feasibility of PP, we need $-\sum_{i \in I} \bar{d}_{i}<\Delta g$, i.e., the change in supply does not exceed the capability of change in load, which is true if a large enough number of loads participate in the load control scheme. We make the following two assumptions on the disutility functions $D_{i}$.

Assumption 1: For $i \in V, D_{i}$ is increasing, strictly convex and twice continuously differentiable over $\left[0, \bar{d}_{i}\right]$.

Assumption 2: For $i \in V$, there exists $\alpha_{i}>0$ so that $D_{i}^{\prime \prime}\left(\Delta d_{i}\right) \geq 1 / \alpha_{i}$ for $\Delta d_{i} \in\left[0, \bar{d}_{i}\right]$.

By [10, Section II-B], we formulate the dual problem of PP as follows:

$$
\max _{p \in \mathbb{R}} \Psi(p):=\sum_{i=1}^{N} \Psi_{i}(p)-p \Delta g
$$

where

$$
\Psi_{i}(p):=\min _{\Delta d_{i} \in\left[0, \bar{d}_{i}\right]} D_{i}\left(\Delta d_{i}\right)-p \Delta d_{i} .
$$

Under Assumption 1, given $p \in \mathbb{R}$, the problem

$$
\min _{\Delta d_{i} \in\left[0, \bar{d}_{i}\right]} D_{i}\left(\Delta d_{i}\right)-p \Delta d_{i}
$$

has a unique minimizer, denoted by

$$
\Delta d_{i}(p)=\min \left\{\max \left\{\left(D_{i}^{\prime}\right)^{-1}(p), 0\right\}, \bar{d}_{i}\right\} .
$$

If $p^{*}$ is an optimal point of the dual problem in (3), then $\left[\Delta d_{1}\left(p^{*}\right), \ldots, \Delta d_{N}\left(p^{*}\right)\right]$ is an optimal point of the primal problem PP. It is easy to show that for any given $p$ and $\bar{p}$ such that $\underline{p} \leq \min _{i} D_{i}^{\prime}(0)$ and $\bar{p} \geq \max _{i} D_{i}^{\prime}\left(\bar{d}_{i}\right)$, the problem in (3) has at least one optimal point $p^{*} \stackrel{i}{\in}[p, \bar{p}]$. So we constrain $p$ to the bounded interval $[p, \bar{p}]$. This helps the convergence proof of Algorithm 1 below. Therefore, the load control scheme focuses on solving the following problem

$$
\mathbf{D P}\left\{\max _{p \in[\underline{p}, \bar{p}]} \Psi(p)=\sum_{i=1}^{N} \Psi_{i}(p)-p \Delta g .\right.
$$

\footnotetext{
${ }^{1}$ In experiments, if $\Delta g(t)$ varies slowly compared to the convergence time of the load control algorithm, as in Section $\mathrm{V}$, the change in load $\Delta d(t)$ tracks $\Delta g(t)$ with minimum global disutility.
} 


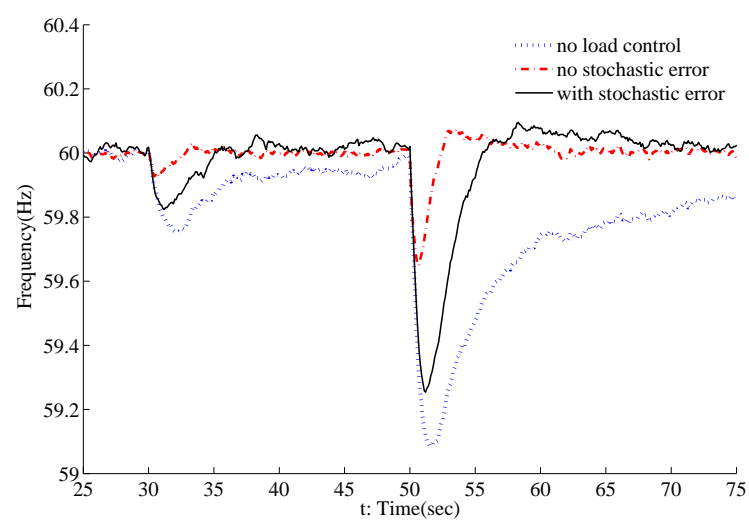

Fig. 2. Frequency response with the scheme proposed in [10]. The dotted line corresponds to that without load control, and the dash-dot line and the solid line respectively correspond to the case where there are no errors and there are stochastic errors in frequency measurement.

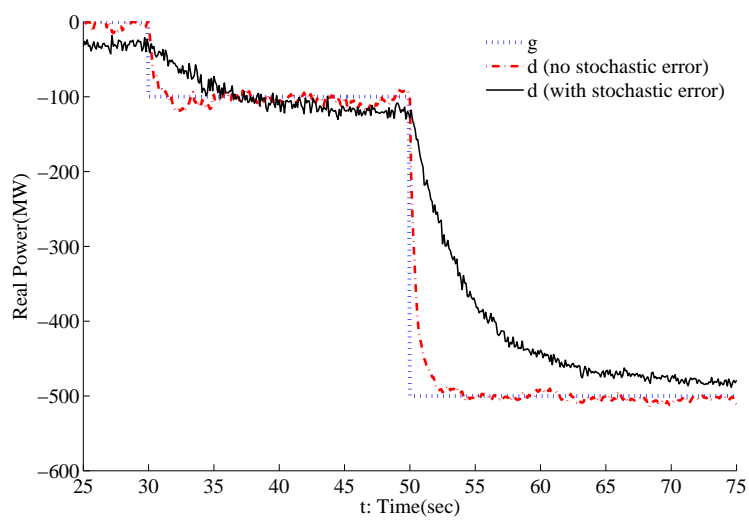

Fig. 3. Total change in load with the scheme proposed in [10]. The dotted line corresponds to the change in supply $\Delta g(t)$, and the dash-dot line and the solid line respectively correspond to the change in load $\Delta d(t)$ when there are no errors and there are stochastic errors in frequency measurements.

The dual variable $p$ in DP is a control signal. In previous works on demand response on a slow timescale, a central coordinator calculates $p$ and broadcasts it to all the loads [14],[15]. To decentralize and speed up the load control so that it does not rely on the load-coordinator communication, in [10] we proposed a decentralized frequency-based load control scheme. Each load independently maintains and updates a value of $p$ using its local frequency measurement. If there are no errors in frequency measurements, the values of $p$ at all loads agree with each other and finally converge to $p^{*}$, the optimal point of DP. However, when there are stochastic frequency measurement errors, the scheme in [10] may perform poorly, as shown in Fig. 2 and 3. The figures respectively compare the frequency response and the change in load under two sudden generation loss events with and without stochastic frequency measurement errors. We see that with stochastic frequency measurement errors, the undershoot in frequency grows almost as large as that without load control, and there is large delay and obvious steady state error between the change in load and the change in supply. This poor performance may be due to the inconsistency between the values of $p$ maintained at different loads, which is caused by the different realizations of the stochastic errors at different loads. In Section IV we will propose a scheme based on inter-load communication to address this issue.

\section{ESTIMATING DEMAND-SUPPLY MISMATCH}

As is presented in [10], the way to decentralize the load control is to update the value of $p$ maintained by each load using $p(t+1)=p(t)+\gamma u_{t}$, where $\gamma>0$ is some stepsize and $u_{t}$, the total demand-supply mismatch at time $t$, is essentially the gradient of the dual objective function $\Psi$. Therefore, it is important to estimate $u_{t}$ from local frequency measurement. Since $u_{t}$ is the input of the power system given by (1), we call the mechanism of estimating $u_{t}$ the input estimator. We now introduce the input estimator with stochastic process disturbance and frequency measurement errors.

With the frequency measurements $\Delta \bar{\omega}_{i, 1}, \ldots, \Delta \bar{\omega}_{i, t}$, load $i$ estimates $u_{0}, \ldots, u_{t-1}$ with the input estimator. The system is given in the form of (1). Use $\hat{x}_{t \mid s}^{i}$ and $\hat{u}_{t \mid s}^{i}$ respectively to denote the estimate of the state $x_{t}$ and that of the input $u_{t}$ with measurements up to time $s$. Starting from $\hat{x}_{0 \mid-1}^{i}$, the input estimator of load $i$ is given recursively by

$$
\begin{gathered}
\hat{u}_{t-1 \mid t}^{i}=M\left(\Delta \bar{\omega}_{i, t}-C \hat{x}_{t \mid t-1}^{i}\right), \\
\hat{x}_{t \mid t}^{i}=\hat{x}_{t \mid t-1}^{i}+B \hat{u}_{t-1 \mid t}^{i}, \\
\hat{x}_{t+1 \mid t}^{i}=A \hat{x}_{t \mid t}^{i},
\end{gathered}
$$

where $M=(C B)^{-1}$ is a scalar under the assumption $C B \neq 0$ mentioned in Section II-A. The input estimator is essentially the filter proposed by Kitanidis [11], which gives unbiased and minimum variance estimate of the state and the input. The covariance of $x_{t \mid t}^{i}$, denoted by $\Sigma_{t \mid t}^{i} \in \mathbb{R}^{n \times n}$, is given recursively by

$$
\begin{aligned}
\Sigma_{t+1 \mid t+1}^{i} & =\left(I_{n}-B M C\right)\left(A \Sigma_{t \mid t}^{i} A^{T}+Q\right)\left(I_{n}-B M C\right)^{T} \\
& +B M W M^{T} B^{T},
\end{aligned}
$$

where $Q$ and $W$ are defined in (2). Denote the error between the input estimate and the real input by $e_{t}^{i}:=\hat{u}_{t \mid t+1}^{i}-u_{t}$. Define the $\sigma$-algebra $F_{t-1}:=\sigma\left(e_{\tau-1}^{i} ; i \in V, 1 \leq \tau \leq t\right)$, which is the history of the input estimate errors before time $t$ for all loads in $V$. By [11], the expectation and the variance of $e_{t}^{i}$ conditioning on $F_{t-1}$ are given by

$$
\mathbb{E}\left(e_{t}^{i} \mid F_{t-1}\right)=0,
$$

and

$$
\mathbb{E}\left[\left(e_{t}^{i}\right)^{2} \mid F_{t-1}\right]=\frac{C A \Sigma_{t \mid t}^{i} A^{T} C^{T}+W}{(C B)^{2}} .
$$

The following proposition provides a condition under which $\mathbb{E}\left[\left(e_{t}^{i}\right)^{2} \mid F_{t-1}\right]$ converges to some constant as $t \rightarrow \infty$.

Proposition 1: Denote the eigenvalues of $\left(I_{n}-B(C B)^{-1} C\right) A \in \mathbb{R}^{n \times n}$ by $\lambda_{s}, s=1, \ldots n$. If $\left|\lambda_{s}\right|<1$ for all $s$, then

$$
\lim _{t \rightarrow \infty} \mathbb{E}\left[\left(e_{t}^{i}\right)^{2} \mid F_{t-1}\right]=\sigma^{2}
$$


where $\sigma^{2}$ is some constant determined by $A, B, C, Q$ and $W$, and independent of $i$.

Proof: Recall that $M=(C B)^{-1}$. The matrix $\left(I_{n}-\right.$ $B M C) Q\left(I_{n}-B M C\right)^{T}+B M W M^{T} B^{T}$ is positive definite. Then by [19, pp. 64], if $\left|\lambda_{s}\right|<1$ for all $s=1, \ldots, n$, the equation

$$
\begin{aligned}
\Sigma & =\left(I_{n}-B M C\right) A \Sigma A^{T}\left(I_{n}-B M C\right)^{T} \\
& +\left(I_{n}-B M C\right) Q\left(I_{n}-B M C\right)^{T}+B M W M^{T} B^{T}
\end{aligned}
$$

has a unique, positive definite solution $\Sigma^{*}$. Additionally, $\lim _{t \rightarrow \infty} \Sigma_{t \mid t}^{i}$ exists and is $\Sigma^{*}[19$, pp. 66]. By (12), we have

$$
\lim _{t \rightarrow \infty} \mathbb{E}\left[\left(e_{t}^{i}\right)^{2} \mid F_{t-1}\right]=\frac{C A \Sigma^{*} A^{T} C^{T}+W}{(C B)^{2}},
$$

where the right hand side is independent of $i$, and can be determined by $A, B, C, Q$ and $W$.

For any system given in the form of (1), we can check whether the condition in Proposition 1 is satisfied. As a partial justification, it holds for many power system models and in particular, those in Section V. The implication of this condition needs future study. To prove the convergence of the algorithm proposed later, we need to bound the variance of the input estimate error. The following corollary, which is a straightforward consequence of Proposition 1, gives such a bound.

Corollary 1: If the condition for Proposition 1 holds, then $\mathbb{E}\left[\left(e_{t}^{i}\right)^{2} \mid F_{t-1}\right] \leq \bar{\sigma}^{2}$ for all $i \in V$ and all $t \geq 0$, where $\bar{\sigma}^{2}$ is some constant determined by $A, B, C, Q, W$ and the initial covariance $\Sigma_{0 \mid 0}^{i}$ of all $i \in V$.

With the input estimator and its property introduced above, we can propose the load control algorithm and prove its convergence.

\section{LOAD CONTROL ALGORITHM}

In this section, we first introduce the load control algorithm that solves the optimization problem PP in a decentralized way. Then we discuss the communication architecture that supports the information exchange between loads. Finally we present the main result regarding the convergence of the proposed algorithm.

\section{A. Decentralized load control algorithm}

As is discussed in Section II-B, in the decentralized load control with stochastic frequency measurement errors, there is inconsistency between the values of $p$ maintained by individual loads. To resolve such inconsistency, we propose a new algorithm based on inter-load communication. We define the set of loads that communicate directly with load $i$ to be $\mathscr{N}(i)$. At each time $t$, load $i$ assigns a weight $r_{i j}(t)$ for all $j \in \mathscr{N}(i)$, and a weight $r_{i i}(t)$ for itself. Conditions on the weights will be discussed later in Section IV-B. The load control algorithm is presented as follows.

\section{Algorithm 1: Decentralized load control algorithm}

At time $t=0$, the following information is given to all the loads $i \in V$ : the system model which contains matrices $A, B$ and $C$, the lower bound $p$ and upper bound $\bar{p}$ that is defined in Section II-B, and a sequence of positive stepsize $\left\{\gamma_{t}, t=\right.$ $1,2, \ldots\}$ which is the same for all loads.
Each load $i$ starts from arbitrary initial state estimate $\hat{x}_{1 \mid 0}^{i}$ and initial value of dual variable $q_{i}(0)$.

At times $t=1,2, \ldots$, load $i$ :

1) Measures the frequency deviation $\Delta \bar{\omega}_{i, t}$, and calculates $\hat{u}_{t-1 \mid t}^{i}$ using the input estimator (7)-(9).

2) Updates the dual variable according to

$$
p_{i}(t)=\max \left\{\min \left\{q_{i}(t-1)+\gamma_{t} \hat{u}_{t-1 \mid t}^{i}, \bar{p}\right\}, \underline{p}\right\},
$$

and transmits $p_{i}(t)$ to all loads $j \in \mathscr{N}(i)$.

3) Receives the value of $p_{j}(t)$ from all $j \in \mathscr{N}(i)$, and calculates

$$
q_{i}(t)=\sum_{j=i, j \in \mathscr{N}(i)} r_{i j}(t) p_{j}(t) .
$$

4) Computes the change in its load

$$
\Delta d_{i}(t)=\Delta d_{i}\left(q_{i}(t)\right),
$$

where $\Delta d_{i}(\cdot)$ is defined in (5).

Remark 1: Algorithm 1 is similar to the stochastic gradient projection method proposed in [18]. By definition of $\Psi_{i}(\cdot)$ and $\Delta d_{i}(\cdot)$ in Section II-B, we have

$$
\Psi_{i}^{\prime}\left(q_{i}(t-1)\right)=-\Delta d_{i}\left(q_{i}(t-1)\right) .
$$

Then, by (15),(17) and the definition of $u_{t}$ and $e_{t}^{i}$, the iteration of $p_{i}(t)$ can be written as

$$
p_{i}(t)=\left[q_{i}(t-1)+\gamma_{t}\left(\sum_{j=1}^{N} \Psi_{j}^{\prime}\left(q_{j}(t-1)\right)-\Delta g+e_{t-1}^{i}\right)\right]_{\underline{p}}^{\bar{p}},
$$

where $[\cdot]_{p}^{\bar{p}}:=\max \{\min \{\cdot, \bar{p}\}, \underline{p}\}$. It takes the similar form with [18, Equation (2.3)]. However, instead of using the gradient of load i's local function $\Psi_{i}^{\prime}\left(q_{i}(t-1)\right)$ as the increment in [18], in Algorithm 1 each load can access the summation of the gradients over all the loads, as a result of frequency measurement and input estimation.

\section{B. Inter-load communication}

Before presenting the main result regarding Algorithm 1, we first introduce the communication architecture supporting the information exchange in (16), as well as some conditions on the weights $r_{i j}(t)$ for the convergence proof of Algorithm 1.

Fig. 4 shows the communication architecture in smart grid, proposed by Trilliant [16]. To solve a global optimization problem like PP, a natural idea is to have every load communicate with a central coordinator which calculates a control signal and distributes this signal to all the loads. This approach requires the loads and the coordinator to communicate across both the wide area network (WAN) and the neighborhood area network (NAN), which may involve larger delay than communications purely over NAN ${ }^{2}$. Therefore, we eliminate the central coordinator in Algorithm 1 and take advantage of communications between loads. Such inter-load communications can be performed via NAN, where reliable, scalable,

\footnotetext{
${ }^{2}$ We ignore the home area network (HAN) if we regard each house as an aggregated controllable load and do not consider inter-appliance communications within the house.
} 


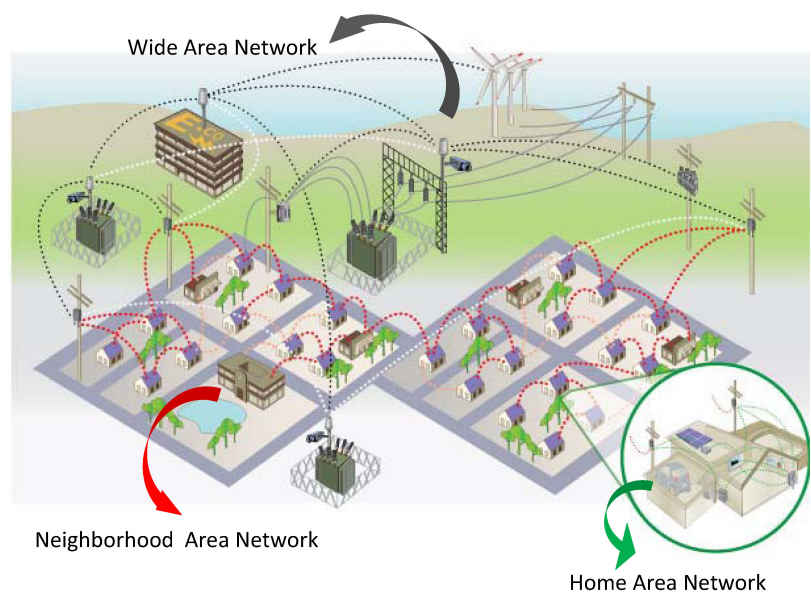

Fig. 4. Communication architecture in smart grid. The home area network (HAN) involves the communication between the household appliances and the smart meter at the house. The neighborhood area network (NAN) aids the communications between the utilities and the smart meters. The wide area network (WAN) is responsible for the distribution, transmission or generation level communications.

fast responding and cost-effective communication technologies such as 802.2.15.4/ZigBee are widely used [16],[17].

Now we describe the rules on the weights $r_{i j}(t)$ by which load $i$ takes the average of information it receives from load $j$. We have following assumptions on these weights.

Assumption 3: There exists a scalar $0<\eta<1$ such that for all $i=1, \ldots, N$ and all $t \geq 0, r_{i j}(t) \geq \eta$ if $j=i$ or $j \in \mathscr{N}(i)$, and $r_{i j}(t)=0$ otherwise.

Assumption 4: For all $i=1, \ldots, N$ and all $t \geq 0, \sum_{j=1}^{N} r_{i j}(t)=$ 1, and $\sum_{j=1}^{N} r_{j i}(t)=1$.

Note that with Assumption 3, Equation (16) becomes

$$
q_{i}(t)=\sum_{j=1}^{N} r_{i j}(t) p_{j}(t) .
$$

Besides, to make sure the information at each load $j$ will impact load $i$ infinitely often, we require that within any finite period of time, the set of links which have ever been connected form a strongly connected graph. Define $E_{t}:=\left\{(i, j) \mid r_{i j}(t)>0\right\}$ to be the set of connected links at time $t$. The connectivity requirement above is formally stated in the following assumption.

Assumption 5: There exists a integer $Q \geq 1$ such that the graph $\left(V, \underset{\tau=1, \ldots, Q}{\bigcup} E_{t+\tau-1}\right)$ is strongly connected for all $t$.

Define $R(t)$ to be the matrix with $(i, j)$-th entry $r_{i j}(t)$, and define $\Phi(t, s)=R(t) R(t-1) \ldots R(s+1)$. The following result given by [18, Lemma 3.2] will be used in the convergence proof of Algorithm 1:

$$
\left|[\Phi(t, s)]_{i j}-\frac{1}{N}\right| \leq \theta \beta^{t-s},
$$

where

$$
\theta=\left(1-\frac{\eta}{4 N^{2}}\right)^{-2}, \quad \beta=\left(1-\frac{\eta}{4 N^{2}}\right)^{\frac{1}{Q}}
$$

Remark 2: Assumption 5 states a weaker condition than requiring the graph to be strongly connected all the time. It only requires that the union of links which have ever been connected within any $Q$ time intervals to strongly connect the graph. Particularly, the graph is strongly connected all the time if $Q=1$. The smaller $Q$ is, the stronger connectivity the graph has, and the better performance we expect the control to be. This corresponds to (21), where the smaller $Q$ is, the smaller $\beta$ is, the faster $\Phi(t, s)$ converges to the matrix $\frac{1}{N} 11^{T}$ and the better performance we can expect.

\section{Convergence of Algorithm 1}

Now we present the main results regarding the convergence of Algorithm 1. We first consider the case where the stepsize sequence $\left\{\gamma_{t}, t=1,2, \ldots\right\}$ converges to some nonnegative constant. Theorem 1 gives a bound between the maximum expected value of the dual objective function $\Psi$ and the optimal value of DP, denoted by $\Psi^{*}$.

Theorem 1: Suppose Assumptions 1-5 hold. If $\lim _{t \rightarrow \infty} \gamma_{t}=\gamma \geq$ 0 and $\sum_{t=1}^{\infty} \gamma_{t}=\infty$, then, for all $i \in V$,

$$
\begin{aligned}
\limsup _{t \rightarrow \infty} \mathbb{E}\left[\Psi\left(p_{i}(t)\right)\right] & \geq \Psi^{*}-\frac{\gamma\left(G^{2}+\bar{\sigma}^{2}\right)}{2} \\
& -\gamma G(\bar{\alpha} N L+G)\left(2+\frac{N \theta \beta}{1-\beta}\right),
\end{aligned}
$$

where $G:=\max \left\{\left|\sum_{i=1}^{N} \bar{d}_{i}+\Delta g\right|,|\Delta g|\right\}$, and $L:=\bar{p}-\underline{p}$. Recall that $\bar{\alpha}=\max _{i \in V} \alpha_{i}$, with $\alpha_{i}$ defined in Assumption 2, and $\bar{\sigma}^{2}$ is defined in Corollary 1.

Proof: See Appendix A.

By taking $\gamma=0$ in (22), it implies that when the stepsize $\gamma_{t}$ diminishes with increasing $t$, the maximum expected value of $\Psi$ achieved by Algorithm 1 is exactly the optimal value of the dual problem DP.

Corollary 2: Suppose Assumptions 1-5 hold. If $\lim _{t \rightarrow \infty} \gamma_{t}=0$ and $\sum_{t=1}^{\infty} \gamma_{t}=\infty$, then, for all $i \in V$,

$$
\limsup _{t \rightarrow \infty} \mathbb{E}\left[\Psi\left(p_{i}(t)\right)\right]=\Psi^{*} .
$$

By using further relatively reasonable restrictions on the stepsize $\gamma_{t}$, the sequence generated by each load using Algorithm 1 almost surely converges to the same optimal point of the dual problem DP. In addition, the change in load $\Delta d_{i}(t)$ almost surely converges to the solution of the load control optimization problem PP. This result is formally stated in Theorem 2.

Theorem 2: Suppose Assumptions 1-5 hold. When $\sum_{t} \gamma_{t}=\infty$ and $\sum_{t=1}^{\infty} \gamma_{t}^{2}<\infty$, the sequence $\left\{q_{i}(t), t=1,2, \ldots\right\}$ of each load $i \in V$ converges to the same optimal point of the dual problem DP with probability 1 and in mean square. Moreover, define $\Delta d(t)=\left[\Delta d_{1}(t), \ldots, \Delta d_{N}(t)\right]^{T}$. Then the sequence $\{\Delta d(t), t=$ $1,2, \ldots\}$ converges to the optimal point of the primal problem PP with probability 1 . 
Proof: With Assumptions 1-5, Equation (11) and Corollary 1, all the conditions for [18, Theorem 6.2] are satisfied. We follow the same technique and get that for all $i \in V$, the sequence $\left\{q_{i}(t), t=1,2, \ldots\right\}$ converges to the same optimal point of the dual problem DP with probability 1 and in mean square. Define $\Delta d(q(t))=\left[\Delta d_{1}\left(q_{1}(t)\right), \ldots, \Delta d_{N}\left(q_{N}(t)\right)\right]^{T}$. By the analysis in [10, Section II-B], the sequence $\{\Delta d(q(t)), t=$ $1,2, \ldots\}$ converges to the optimal point of the primal problem $\mathbf{P P}$ with probability 1 . Then we can get the result of Theorem 2 since $\Delta d(t)=\Delta d(q(t))$ by (17).

\section{CAse studies}

In this section, we use relatively detailed power system models to evaluate the performance of Algorithm 1 when there are stochastic frequency measurement errors at the loads. We observe the frequency response, the global end-use disutility and how the change in load follows the change in supply under sudden generation loss events in the system. With numerical experiments, we test the robustness of the proposed scheme to modeling inaccuracies when the loads use a simplified power system model instead of the accurate one. We also discuss the tradeoff between communication effort and the performance of the load control.

\section{A. System settings}

We use the power system model which has the same block diagram, transfer functions and parameters as $[10$, Section $\mathrm{V}-\mathrm{A}]$. We do not show the details here due to space limitation.

The system under consideration is a per unit system with baseline power $P_{\text {base }}=2000$ MVA. The sampling time is $\Delta t=0.1 \mathrm{~s}$. We take $N=101$ loads, which are connected in a graph where each load is placed on a ring and has links to precisely $K$ previous neighbors and $K$ subsequent neighbors, for $0 \leq K \leq(N-1) / 2$. Load $i \in V=\{1,2, \ldots, N\}$ has a disutility function $D_{i}\left(\Delta d_{i}\right)=\left(\Delta d_{i}\right)^{2} /\left(2 \bar{\alpha}_{i}\right)$, where $\bar{\alpha}_{i}$ is a random positive number, e.g., uniformly distributed on $[1,3]$ in our experiments. For $i \in V, \Delta d_{i} \in\left[0, \bar{d}_{i}\right]$, where $\bar{d}_{i}$ is a positive random number and $\sum_{i \in V} \bar{d}_{i}=0.30$ per unit (pu). We use a diminishing stepsize $\gamma_{t}=\gamma_{0} / t$ for some arbitrarily selected $\gamma_{0}>0$, so the stepsize satisfies $\sum_{t=1}^{\infty} \gamma_{t}=\infty$ and $\sum_{t=1}^{\infty} \gamma_{t}^{2}<\infty$. Therefore, all the conditions in Theorems 1 and 2 are satisfied.

The unscheduled change in real power supply $\Delta g(t)$ contains two step changes to resemble generation loss events. It is initially zero. At time $t=2 \mathrm{~s}$, the first step change occurs with $\Delta g(t)$ falling by $0.05 \mathrm{pu}$; at $t=20 \mathrm{~s}$, the second step change occurs with $\Delta g(t)$ falling further by $0.20 \mathrm{pu}$. The stochastic process disturbance $\zeta_{t}$ and frequency measurement error $\xi_{t}^{i}$ are as described in Section II-A, with variance $Q=$ $B(0.01 \mathrm{pu})^{2} B^{T}$ for the $B$ determined by the given parameters and $W=(0.005 \mathrm{pu})^{2}$.

\section{B. Performance and robustness to modeling inaccuracies}

Besides observing the performance of the fast load control with Algorithm 1 when all loads have the accurate, high-order system model given by matrices $A, B$ and $C$, we also show the performance of using a simplified, second-order model on the

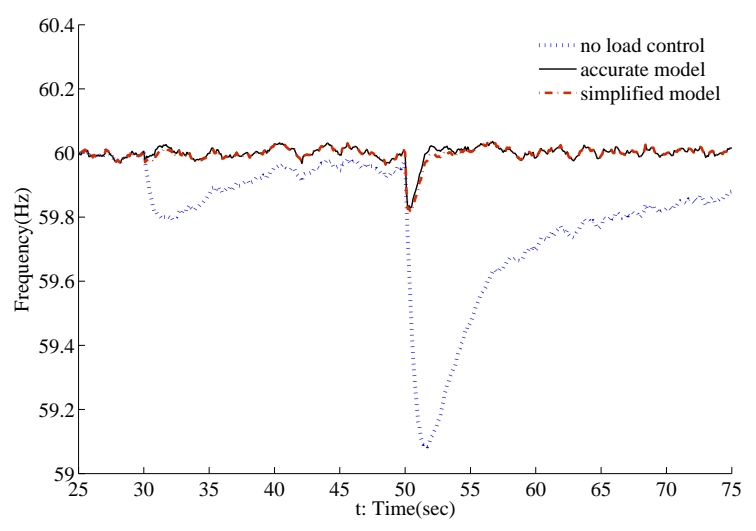

Fig. 5. Frequency reponse with fast load control using different models on the load side. The solid line and the dash-dot line are respectively the frequency response with the loads using the accurate model and the simplified model. The dotted line is that without load control.

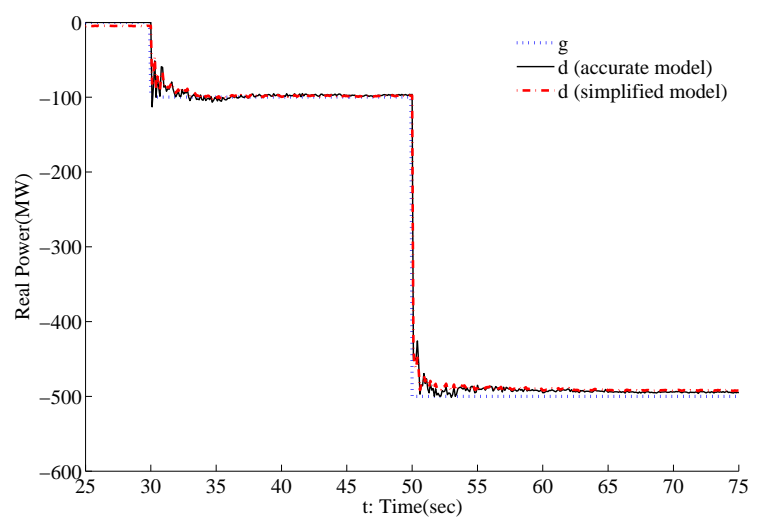

Fig. 6. Total change in load with fast load control using different models on the load side. The dotted line is the change in supply $\Delta g(t)$. The solid line and the dash-dot line are respectively the total change in load with the loads using the accurate model and the simplified model.

load side. This is due to the practical consideration that the utility company may not reveal the exact system information to the loads for privacy considerations, and the accurate model may be so complicated that it costs much to determine it, deliver it to loads and compute with it. The detailed form of the simplified model and the way to derive it are presented in [10, Section V-B].

Fig. 5-7 respectively show the frequency response, the total change in load and the global end-use disutility with the loads using the accurate model or the simplified model, all with $K=10$ in the communication graph. We see that all the results given by using the simplified model are quite close to those by using the accurate model. They can both drive the frequency back to around $60 \mathrm{~Hz}$, make the load following the supply and minimize the disutility within several seconds, much faster than AGC. This also implies the proposed scheme is robust to modeling inaccuracies.

\section{Tradeoff between communication and performance}

Now we discuss the tradeoff between inter-load communication and the performance of load control. In the communi- 


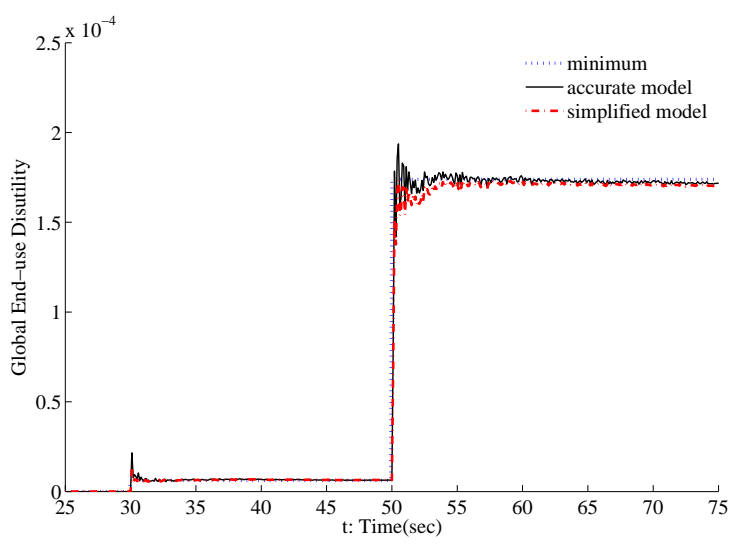

Fig. 7. Global end-use disutility with fast load control using different models on the load side. The dotted line is the minimal possible disutility. The solid line and the dash-dot line are respectively the disutility with the loads using the accurate model and the simplified model.

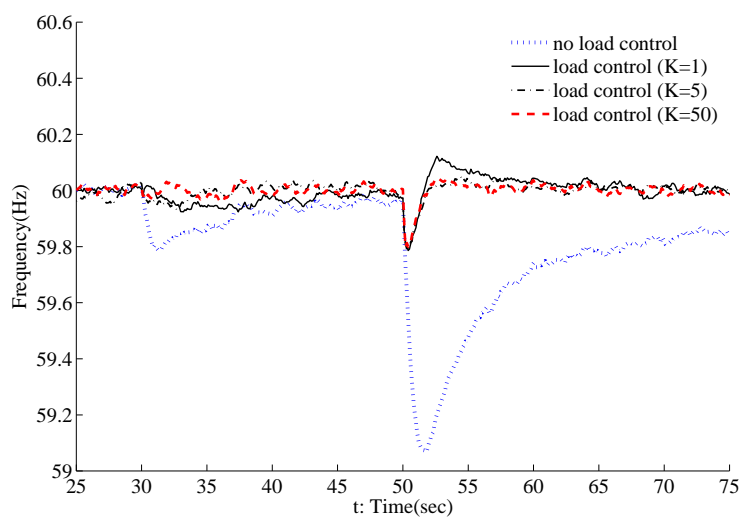

Fig. 8. Frequency reponse with different connectivity in the communication graph. The dotted line is the frequency response without load control. The solid line, the dash-dot line and the dashed line respectively correspond to the case $K=1, K=5$ and $K=50$.

cation graph we used, the larger $K$ is, the more strongly the loads are connected. Fig. 8-10 respectively show the frequency response, the total change in load and the global end-use disutility with $K=1,5,50$. Note that $K=1$ is the weakest time-invariant connection that satisifies Assumption 5, and $K=50$ is the strongest connection and forms a complete graph.

Comparing Fig. 8-10 with Fig. 5-7 (in which $K=10$ ) and Fig. 2-3 (in which there is no communication), we see that with stronger connectivity and more communciation effort, the load control performs better in the sense that there is smaller undershoot in frequency response, shorter transient time before convergence and smaller steady state error in minimizing disutility and following the supply. On the other hand, the most distinguishable results occur between no communication and $K=1$, which implies even the weakest inter-load communication improves the results significantly. When $K$ is larger than 5 , the results are not so distinguishable with increasing $K$. It implies that the proposed load control scheme can address the stochastic frequency measurement effectively using a moderate amount of communication via the neighborhood area network.

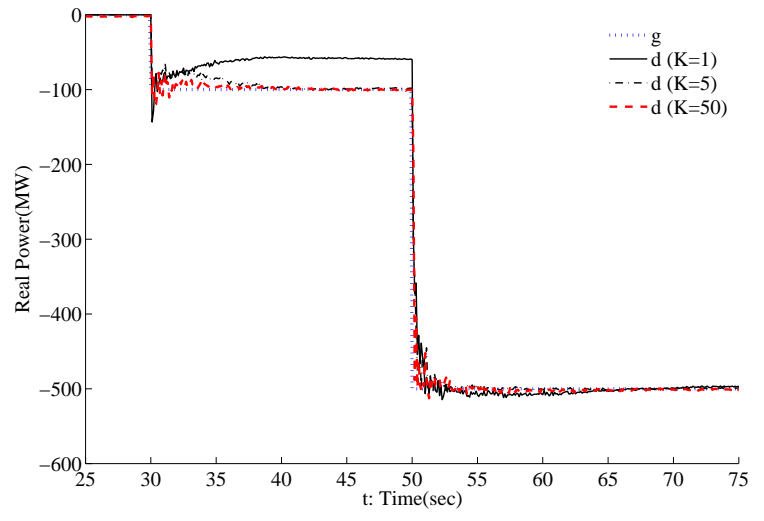

Fig. 9. Total change in load with different connectivity in the communication graph. The dotted line is the change in supply $\Delta g(t)$. The solid line, the dashdot line and the dashed line are respectively the change in load in the case $K=1, K=5$ and $K=50$.

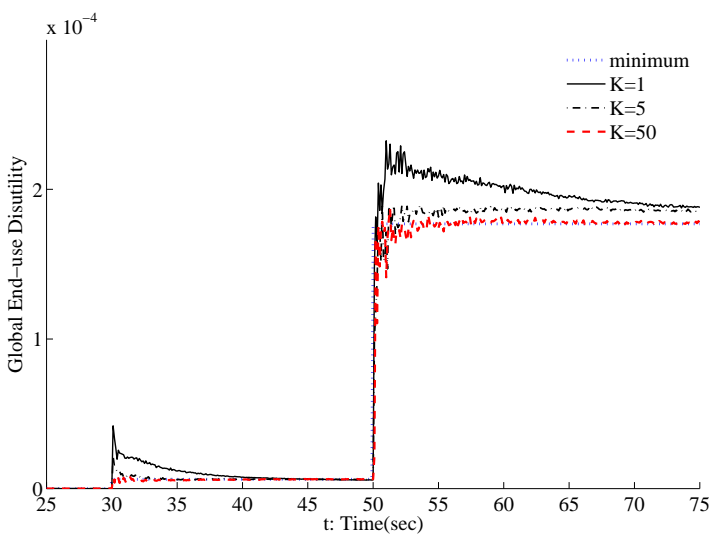

Fig. 10. Global end-use disutility with different connectivity in the communication graph. The dotted line is the minimal possible disutility. The solid line, the dash-dot line and the dashed line respectively correspond to the case $K=1, K=5$ and $K=50$.

\section{CONCLUSIONS AND FUTURE WORK}

In this paper, we propose a fast load control scheme with stochastic frequency measurement errors in the power system. The scheme aims to match the real-time demand with supply and regulate the frequency while minimizing the global enduse disutility. The loads estimate the global demand-supply mismatch from local frequency measurements and accordingly adjust their power consumption level in a decentralized way. To address the estimation errors caused by stochastic frequency measurements, inter-load communication via the neighborhood area network is used. The convergence of the proposed scheme has been proved. Experiments show that the proposed scheme can make the change in load follow the change in supply and restore frequency around nominal value on a timescale faster than AGC. Moreover, the scheme performs well even when the loads use a simplified power system model instead of the accurate model. We discuss the tradeoff between communication effort and the performance of load control. Experimental results indicate that a moderate amount of inter-load communication can improve performance 
significantly under stochastic frequency measurement errors.

Currently the fast load control scheme is based on a strongly connected area in the power system which contains the dynamics of a single synchronous machine. In the future, we will work on the power system composed of multiple interconnected areas with multiple synchronous machines and asynchronism between areas. In that case, the external input for each area, denoted by $\Delta g(t)$ in this paper, becomes the inter-area tie-line power which depends on the multiple dynamics, the control scheme within each area and the coordinations between areas. Distributed algorithm and communication requirement under the multi-area settings remain to be explored.

\section{APPENDIX A}

\section{PROOF OF THEOREM 1}

We first show two lemmas as a preparation for proving Theorem 1. Define $y(t):=\frac{1}{N} \sum_{i=1}^{N} p_{i}(t)$. Lemma 1 bounds the error between $y(t)$ and $p_{i}(t)$, i.e., the inconsistency of information between loads.

Lemma 1: Suppose Assumptions 3, 4 and 5 hold. Then for all $i \in V$ and $t \geq 0$,

$$
\begin{aligned}
\left|y(t)-p_{i}(t)\right| & \leq \theta \beta^{t} \sum_{j=1}^{N}\left|p_{j}(0)\right|+\theta \sum_{\tau=1}^{t-1} \gamma_{\tau} \beta^{t-\tau} \sum_{j=1}^{N}\left(G+\left|e_{\tau-1}^{j}\right|\right) \\
& +\frac{\gamma_{t}}{N} \sum_{j=1}^{N}\left(G+\left|e_{t}^{j}\right|\right)+\gamma_{t}\left(G+\left|e_{t}^{i}\right|\right)
\end{aligned}
$$

where $G=\max \left\{\left|\sum_{i=1}^{N} \bar{d}_{i}+\Delta g\right|,|\Delta g|\right\}$, and $\theta, \beta$ are defined in (21).

Proof: By (18) and the fact that $\Delta d_{i}(p) \in\left[0, \bar{d}_{i}\right]$ for all $p \in \mathbb{R}$, we have

$$
\begin{aligned}
\left|\sum_{j=1}^{N} \Psi_{j}^{\prime}\left(q_{j}(t-1)\right)-\Delta g\right| & =\left|-\sum_{j=1}^{N} \Delta d_{i}\left(q_{j}(t-1)\right)-\Delta g\right| \\
& \leq \max \left\{\left|\sum_{i=1}^{N} \bar{d}_{i}+\Delta g\right|,|\Delta g|\right\}=G .
\end{aligned}
$$

Then, following the technique of [18, Lemma 4.1], we get the result of Lemma 1.

Now we derive a bound on the distance between $q_{i}(t)$ and any $x \in[p, \bar{p}]$. This bound is related to $\left|y(t)-p_{i}(t)\right|$ and the difference of the dual objective function $\Psi(y(t))-\Psi(x)$.

Lemma 2: Suppose Assumptions 1-5 hold. Then for all $t \geq$ 0 and any $x \in[p, \bar{p}]$, we have

$$
\begin{aligned}
\sum_{i=1}^{N}\left(q_{i}(t+1)-x\right)^{2} & \leq \sum_{i=1}^{N}\left(q_{i}(t)-x\right)^{2} \\
& +2 \gamma_{t+1} N(\Psi(y(t))-\Psi(x)) \\
& +2 \gamma_{t+1} N \bar{\alpha} L \sum_{i=1}^{N}\left|y(t)-p_{i}(t)\right| \\
& +2 \gamma_{t+1} \sum_{i=1}^{N} e_{t}^{i}\left(q_{i}(t)-x\right)+\gamma_{t+1}^{2} \sum_{i=1}^{N}\left(G+\left|e_{t}^{i}\right|\right)^{2}
\end{aligned}
$$

where $\bar{\alpha}:=\max _{i \in V} \alpha_{i}$, with $\alpha_{i}$ defined in Assumption 2, and $L=\bar{p}-p$.

Proof: By (19) and the convexity of squared norm, we have

$$
\begin{aligned}
\sum_{i=1}^{N}\left(q_{i}(t+1)-x\right)^{2} & =\sum_{i=1}^{N}\left(\sum_{j=1}^{N} r_{i j}(t+1) p_{j}(t+1)-x\right)^{2} \\
& \leq \sum_{i=1}^{N} \sum_{j=1}^{N} r_{i j}(t+1)\left(p_{j}(t+1)-x\right)^{2} \\
& =\sum_{j=1}^{N}\left(p_{j}(t+1)-x\right)^{2}
\end{aligned}
$$

Besides, by (??), and using the projection property, we have

$$
\begin{aligned}
\left(p_{i}(t+1)-x\right)^{2} & \leq\left(q_{i}(t)+\gamma_{t+1}\left(\sum_{j=1}^{N} \Psi_{j}^{\prime}\left(q_{j}(t)\right)-\Delta g+e_{t}^{i}\right)-x\right)^{2} \\
& =\left(q_{i}(t)-x\right)^{2} \\
& +2 \gamma_{t+1}\left(\sum_{j=1}^{N} \Psi_{j}^{\prime}\left(q_{j}(t)\right)-\Delta g\right)\left(q_{i}(t)-x\right) \\
& +2 \gamma_{t+1} e_{t}^{i}\left(q_{i}(t)-x\right) \\
& +\gamma_{t+1}^{2}\left(\sum_{j=1}^{N} \Psi_{j}^{\prime}\left(q_{j}(t)\right)-\Delta g+e_{t}^{i}\right)^{2} .
\end{aligned}
$$

Recall that

$$
\begin{aligned}
\Psi_{i}^{\prime}(\cdot) & =-\Delta d_{i}(\cdot) \\
& =\min \left\{\max \left\{\left(D_{i}^{\prime}\right)^{-1}(\cdot), 0\right\}, \bar{d}_{i}\right\} .
\end{aligned}
$$

Since $D_{i}$ is twice continuously differentiable by Assumption 1 , we can use the mean value theorem and have

$$
\begin{aligned}
\Psi_{i}^{\prime}\left(q_{i}(t)\right) & =\Psi_{i}^{\prime}(y(t))+\Psi_{i}^{\prime}\left(q_{i}(t)\right)-\Psi_{i}^{\prime}(y(t)) \\
& =\Psi_{i}^{\prime}(y(t))+\Psi_{i}^{\prime \prime}\left(z_{i}(t)\right)\left(q_{i}(t)-y(t)\right),
\end{aligned}
$$

where $z_{i}(t)$ is some value between $q_{i}(t)$ and $y(t)^{3}$. Besides, note that

$$
\begin{aligned}
y(t)=\frac{1}{N} \sum_{i=1}^{N} p_{i}(t) & =\frac{1}{N} \sum_{i=1}^{N} \sum_{j=1}^{N} r_{j i}(t) p_{i}(t) \\
& =\frac{1}{N} \sum_{j=1}^{N} \sum_{i=1}^{N} r_{j i}(t) p_{i}(t)=\frac{1}{N} \sum_{j=1}^{N} q_{j}(t),
\end{aligned}
$$

we have

$$
\begin{aligned}
& \left(\sum_{j=1}^{N} \Psi_{j}^{\prime}\left(q_{j}(t)\right)-\Delta g\right) \sum_{i=1}^{N}\left(q_{i}(t)-x\right) \\
& =\left(\sum_{j=1}^{N} \Psi_{j}^{\prime}(y(t))-\Delta g+\sum_{j=1}^{N} \Psi_{j}^{\prime \prime}\left(z_{j}(t)\right)\left(q_{j}(t)-y(t)\right)\right) \\
& N(y(t)-x) \\
& =N\left(\Psi^{\prime}(y(t))+\sum_{j=1}^{N} \Psi_{j}^{\prime \prime}\left(z_{j}(t)\right)\left(q_{j}(t)-y(t)\right)\right)(y(t)-x)
\end{aligned}
$$

${ }^{3}$ At the point where $\Psi_{i}^{\prime}$ is not differentiable, the $\Psi_{i}^{\prime \prime}$ in (27) should be replaced by the subgradient of $\Psi_{i}^{\prime}$, without influncing the proof. 
By $(25)(26)(28)$, we have

$$
\begin{aligned}
& \sum_{i=1}^{N}\left(q_{i}(t+1)-x\right)^{2} \leq \sum_{i=1}^{N}\left(q_{i}(t)-x\right)^{2} \\
& +2 \gamma_{t+1} N\left(\Psi^{\prime}(y(t))+\sum_{j=1}^{N} \Psi_{j}^{\prime \prime}\left(z_{j}(t)\right)\left(q_{j}(t)-y(t)\right)\right)(y(t)-x) \\
& +2 \gamma_{t+1} \sum_{i=1}^{N} e_{t}^{i}\left(q_{i}(t)-x\right) \\
& +\gamma_{t+1}^{2} \sum_{i=1}^{N}\left(\sum_{j=1}^{N} \Psi_{j}^{\prime}\left(q_{j}(t)\right)-\Delta g+e_{t}^{i}\right)^{2} .
\end{aligned}
$$

By the concavity of the dual objective function $\Psi$, we have

$$
\Psi^{\prime}(y(t))(y(t)-x) \leq \Psi(y(t))-\Psi(x) .
$$

By Assumption 2 and the fact that $\Psi_{i}^{\prime}(\cdot)=-\Delta d_{i}(\cdot)=$ $\min \left\{\max \left\{\left(D_{i}^{\prime}\right)^{-1}(\cdot), 0\right\}, \bar{d}_{i}\right\}$, we have that

$$
\Psi_{j}^{\prime \prime}\left(z_{j}(t)\right) \leq \alpha_{i} \leq \bar{\alpha}
$$

In addition, since $y(t)$ is a convex combination of $p_{i}(t)$, and $p_{i}(t) \in[p, \bar{p}]$ by the projection, we have $y(t) \in[p, \bar{p}]$. Since $x \in[\underline{p}, \bar{p}]$, then $|y(t)-x| \leq L$ with $L=\bar{p}-\underline{p}$. Combining this and (31), we have

$$
\begin{aligned}
& \left(\sum_{j=1}^{N} \Psi_{j}^{\prime \prime}\left(z_{j}(t)\right)\left(q_{j}(t)-y(t)\right)\right)(y(t)-x) \\
& \leq \bar{\alpha} L \sum_{j=1}^{N}\left|y(t)-q_{j}(t)\right| .
\end{aligned}
$$

Substituting for $q_{j}(t)$ and using the convexity of the absolute value, we have

$$
\begin{aligned}
& \left(\sum_{j=1}^{N} \Psi_{j}^{\prime \prime}\left(z_{j}(t)\right)\left(q_{j}(t)-y(t)\right)\right)(y(t)-x) \\
& \leq \bar{\alpha} L \sum_{i=1}^{N}\left|y(t)-\sum_{j=1}^{N} r_{i j}(t) p_{j}(t)\right| \\
& \leq \bar{\alpha} L \sum_{i=1}^{N} \sum_{j=1}^{N} r_{i j}(t)\left|y(t)-p_{j}(t)\right| \\
& \leq \bar{\alpha} L \sum_{j=1}^{N}\left|y(t)-p_{j}(t)\right| .
\end{aligned}
$$

Moreover, recall that $G$ is the bound on all $u_{t}$, so we have

$$
\begin{aligned}
\left(\sum_{j=1}^{N} \Psi_{j}^{\prime}\left(q_{j}(t)\right)-\Delta g+e_{t}^{i}\right)^{2} & =\left(u_{t}+e_{t}^{i}\right)^{2} \\
& \leq\left(G+\left|e_{t}^{i}\right|\right)^{2}
\end{aligned}
$$

Incorporating (30)(33)(34) into (29), we finally get the result of Lemma 2.

With Lemmas 1 and 2, we can complete the proof of Theorem 1.
Take the expectation of (23), considering $\mathbb{E}\left(\left|e_{t}^{i}\right|\right)=0$ by (11). Then we have

$$
\begin{aligned}
\mathbb{E}\left(\left|y(t)-p_{i}(t)\right|\right) & \leq \theta \beta^{t} \sum_{j=1}^{N}\left|p_{j}(0)\right| \\
& +\theta N G \sum_{\tau=1}^{t-1} \gamma_{\tau} \beta^{t-\tau}+2 \gamma_{t} G .
\end{aligned}
$$

By [18, Lemma 3.1(a)] we have $\lim _{t \rightarrow \infty} \sum_{\tau=1}^{t-1} \gamma_{\tau} \beta^{t-\tau}=\frac{\beta \gamma}{1-\beta}$, so

$$
\lim _{t \rightarrow \infty} \mathbb{E}\left(\left|y(t)-p_{i}(t)\right|\right) \leq \gamma G\left(2+\frac{N \theta \beta}{1-\beta}\right) .
$$

Then, in (24), we take $x$ as $p^{*}$, which is an optimal point for the problem DP, and sum over $t=0, \ldots, T-1$. Then we have

$$
\begin{aligned}
& \sum_{i=1}^{N} \mathbb{E}\left[\left(q_{i}(T)-p^{*}\right)^{2}\right] \leq \sum_{i=1}^{N} \mathbb{E}\left[\left(q_{i}(0)-x\right)^{2}\right] \\
& +2 N \sum_{t=0}^{T-1} \gamma_{t+1}\left(\mathbb{E}[\Psi(y(t))]-\Psi^{*}\right) \\
& +2 N \alpha L \sum_{t=0}^{T-1} \gamma_{t+1} \sum_{i=1}^{N} \mathbb{E}\left(\left|y(t)-p_{i}(t)\right|\right) \\
& +N\left(G^{2}+\bar{\sigma}^{2}\right) \sum_{t=0}^{T-1} \gamma_{t+1}^{2} .
\end{aligned}
$$

It follows that

$$
\begin{aligned}
& 2 N \sum_{t=0}^{T-1} \gamma_{t+1}\left\{\mathbb{E}[\Psi(y(t))]-\Psi^{*}+\bar{\alpha} L \sum_{i=1}^{N} \mathbb{E}\left(\left|y(t)-p_{i}(t)\right|\right)\right. \\
& \left.+\frac{\gamma_{t+1}\left(G^{2}+\bar{\sigma}^{2}\right)}{2}\right\} \\
& \geq \sum_{i=1}^{N} \mathbb{E}\left[\left(q_{i}(T)-p^{*}\right)^{2}\right]-\sum_{i=1}^{N} \mathbb{E}\left[\left(q_{i}(0)-x\right)^{2}\right] \\
& \geq-\sum_{i=1}^{N} \mathbb{E}\left[\left(q_{i}(0)-x\right)^{2}\right]
\end{aligned}
$$

Then, with $\lim _{t \rightarrow \infty} \gamma_{t}=\gamma$ and $\sum_{t} \gamma_{t}=\infty$, we have

$$
\begin{aligned}
\limsup _{t \rightarrow \infty} \mathbb{E}[\Psi(y(t))] & \geq \Psi^{*}-\bar{\alpha} L \sum_{i=1}^{N} \limsup _{t \rightarrow \infty} \mathbb{E}\left(\left|y(t)-p_{i}(t)\right|\right) \\
& -\frac{\gamma\left(G^{2}+\bar{\sigma}^{2}\right)}{2} .
\end{aligned}
$$

Note that

$$
\begin{aligned}
\mathbb{E}\left[\Psi\left(p_{i}(t)\right)-\Psi(y(t))\right] & \geq-\mathbb{E}\left[\Psi^{\prime}\left(z_{i}(t)\right)\left(p_{i}(t)-y(t)\right)\right] \\
& \geq-G \mathbb{E}\left(\left|y(t)-p_{i}(t)\right|\right),
\end{aligned}
$$

where $z_{i}(t)$ lies between $p_{i}(t)$ and $y(t)$. Incorpeorating (40) and (36) into (39), we have the result (22).

\section{REFERENCES}

[1] A. Molina-Garcia, F. Bouffard and D. S. Kirschen, "Decentralized demand-side contribution to primary frequency control," IEEE Trans. on Power Systems, vol. 26, no. 1, pp. 411-419, 2001.

[2] M. Ilic and Q. Liu, "Toward sensing, communications and control architectures for frequency regulation in systems with highly variable resources," Control and Optimization Theory for Electric Smart Grids, New York: Springer, 2011. 
[3] D. Trudnowski, M. Donnelly and E. Lightner, "Power-system frequency and stability control using decentralized intelligent loads," in Proc. of the 2005 IEEE T\&D Conf. Expo., Dallas, TX, May 2006.

[4] J. Dong, J. Zuo, L. Wang, K. S. Kook, I. Chung, Y. Liu, et al., "Analysis of power system disturbance based on wide-area frequency measurements," in Proc. of the 2007 IEEE Power and Energy Society General Meeting, Tampa, FL, Jun. 2007.

[5] P. Kundur, Power System Stability and Control, New York: McGraw Hill, Inc., 1994.

[6] D. S. Callaway and I. A. Hiskens, "Achieving controllability of electric loads," Proceedings of the IEEE, vol. 99, no. 1, pp. 184-199, 2011.

[7] A. Brooks, E. Liu, D. Reicher, C. Spirakis and B. Weihl, "Demand dispatch: Using real-time control of demand to help balance generation and load," IEEE Power\&Energy Magazine, vol. 8, no. 3, pp. 21-30, 2010

[8] PNNL, Grid Friendly ${ }^{T M}$ Controller Helps Balance Energy Supply and Demand. [Online]. Available: http://www.gridwise.pnl.gov/docs/pnnlsa36565.pdf.

[9] A. R. Bergen and V. Vittal, Power Systems Analysis, 2nd ed. Upper Saddle River, NJ: Prentice Hall, 2000

[10] C. Zhao, U. Topcu and S. H. Low, "Frequency-based load control in power systems," Technical Report, California Institute of Technology, 2011. [CaltechCDSTR:2011.007]. Available: http://resolver.caltech.edu/CaltechCDSTR:2011.007.

[11] P. K. Kitanidis, "Unbiased minimum-variance linear state estimation," Automatica, vol. 23, no. 6, pp. 775-778, 1987.

[12] S. Gillijns and B. D. Moor, "Unbiased minimum-variance input and state estimation for linear discrete-time systems," Automatica, vol. 43, no. 1, pp. 111-116, 2007.

[13] M. Darouach and M. Zasadzinski, "Unbiased minimum variance estimation for systems with unknown exogenous inputs," Automatica, vol. 33, no. 4, pp. 717-719, 1997.

[14] N. Li, L. Chen and S. H. Low, "Optimal demand response based on utility maximization in power networks," in Proc. of the 2011 IEEE Power and Energy Society General Meeting, Detroit, MI, Jul. 2011.

[15] L. Gan, U. Topcu and S. H. Low, "Optimal decentralized protocols for electric vehicle charging," to appear in Proc. 2011 IEEE Conf. Decision and Control, Orlando, FL, Dec. 2011.

[16] Trilliant Inc., "The Multi-Tier Smart Grid Architecture," [Online]. Available: http://www.trilliantinc.com/solutions/multi-tier-architecture/.

[17] Sonoma Innovation Smart Grid and AMI Technology Consulting, "Smart grid communications architectural framework," Aug. 2009. [Online]. Available: http://www-users.cselabs.umn.edu/classes/Fall2009/seng5861/project/a1ueNt4L.pdf.

[18] S. S. Ram, A. Nedic and V. V. Veeravalli, "Distributed stochastic subgradient projection algorithms for convex optimization," Journal of Optimization Theory and Applications, vol. 147, no. 3, pp. 516-545, 2010.

[19] B. D. O. Anderson and J. B. Moore, Optimal Filtering, New York: Dover Publications, INC., 2005.

[20] P. V. Mieghem, Graph Spectra for Complex Networks, Cambridge, UK: Cambridge University Press, 2011.

[21] D. Bertsekas and J. Tsitsiklis, Parallel and Distributed Computation. Upper Saddle River, NJ: Prentice Hall, 1989.

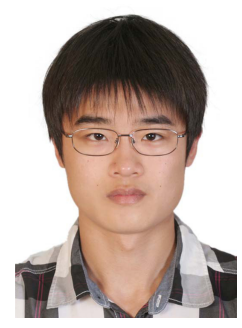

Changhong Zhao Changhong Zhao received his B.S. degree from Tsinghua University, Beijing, China, in automatic control. He is currently working towards a Ph.D. in Electrical Engineering at California Institute of Technology, Pasadena. His main reserach interests are in the distributed control and optimization of networked systems such as smart grid.

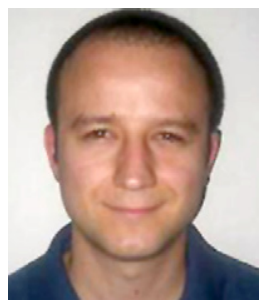

Ufuk Topcu Ufuk Topcu is a postdoctoral scholar in Control and Dynamical Systems at the California Institute of Technology. His research focuses on developing analytical and computational tools that aim to systematically and automatically close the specification-design-verification loop for networked, information-based systems for autonomy, transportation vehicles, and energy networks. He received his $\mathrm{Ph} . \mathrm{D}$. in 2008 from the University of California, Berkeley.

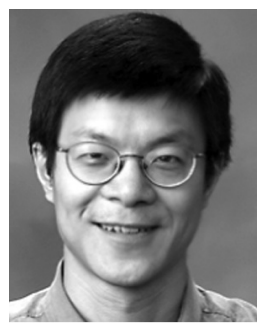

Steven H. Low Steven H. Low received BS from Cornell and $\mathrm{PhD}$ from Berkeley, both in electrical engineering. He is a Professor of the Computing \& Mathematical Sciences and Electrical Engineering departments at Caltech, and hold guest faculty positions with the Swinbourne University, Australia and Shanghai Jiaotong University, China. Prior to that, he was with AT\&T Bell Laboratories, Murray Hill, NJ, and the University of Melbourne, Melbourne, Australia. He was on the Editorial Board of the IEEE/ACM Transactions on Networking, IEEE Transactions on Automatic Control, ACM Computing Surveys, and the Computer Networks Journal. He is currently a Senior Editor of the IEEE Journal on Selected Areas in Communications, and series mentor for its Smart Grid Series. 